\title{
The Structure of Verse: Formal, experimental and computational approaches 19-20 March 2015 Leiden University, The Netherlands
} Varun deCastro-Arrazola

During Thursday the 19th and Friday the 20th of March 2015, a diverse group of about forty scholars gathered in Leiden to discuss issues on verse.

Verse is a wide concept covering poetry, song, chant, nursery rhymes, etc. Some of these activities are considered more related to literature, more to music, more to language, depending on the focus taken by the researcher. As a result, verse studies are scattered across a number of university departments. One of the goals of the workshop was to bring together scholars with different backgrounds but who somehow converge on their interest in verse.

This concern with cutting across disciplines had a particular motivation. The organising committee (Varun deCastro-Arrazola, Teresa Proto, Marc van Oostendorp) is involved in a project investigating how the cognitive capacities universally shared by humans shape cultural systems such as decorative patterns, numeral systems or, in our case, metrical patterns. When dealing with verse within the broader context of cognition, combining a range of approaches proves to be particularly valuable.

Each of the two days of the workshop had its own theme: on Thursday, it was the structure and perception of metred poetry; on Friday, the digital encoding of verse. The first day focused on formal and psychological aspects, while the second day concentrated on computational approaches. Each session was opened by a keynote speaker, followed by a series of seven oral presentations, and rounded off by a general discussion on the subject matter of the day. In addition, there were eight contributions in poster format.

Nigel Fabb, the first invited speaker, launched the event with a paper which discussed the place of the metrical line with respect to working memory. The main claim was that "each line of metrical poetry is constructed and held as a whole unit in working memory capacity." The argument was grounded in a wealth of typological data and a thorough account of the Baddeley-Hitch model of memory. Fabb also recognised that experimental evidence is still lacking from most psychological accounts of verse. Issues like how to define 
an expert poetry listener are some of the obstacles faced by psychological experiments on the topic.

Therefore, most of the contributions on the "structure and perception of metred poetry" were formal accounts based on corpora which offered plausible explanations of how verse is cognitively represented. Some of these accounts rely more on abstract metrical templates (e.g. Jean Louis Aroui's analysis of Classical Arabic Kamil), while others rely almost exclusively on the phonological knowledge of the spoken language (e.g. Tomas Riad's talk on metre as improved phonological prosody). Nonetheless, working with a living tradition, Paolo Bravi could approach the creation of Sardinian improvised poetry from an online perspective, getting closer to the poet's mind while the text is being generated.

The Friday session was opened by Kevin Ryan. Through case studies of Greek, Latin and Sanskrit data, he discussed extensively the problem of evaluating frequencies in verse corpus studies. Phenomena can appear to be under- or over-represented in a certain metrical position, but without proper baselines of what to be expected, these frequencies are hardly interpretable. Statistical analysis of big corpora, a current trend in the humanities, may be more prone to producing raw quantitative results of this kind. However, as Ryan showed, digital data also provides the means to better assess observed frequencies; this can be done, for instance, using prose models or by scrambling techniques.

The strand of work described by Ryan relies on the digitalisation and annotation of corpora, which was the subject matter of several presentations. Projects like ReMetCa (Elena González-Blanco and Clara I. Martínez Cantón) work towards standard and efficient ways of digitally encoding verse material. Still, other projects take some plain digital text as a starting point and develop algorithms to identify basic features such as syllables, and more complex features such as rhyme (Dan Brown) or rhythm (Klemens Bobenhausen and Benjamin Hammerich).

All in all, despite the variety of backgrounds and methodologies present, the workshop benefitted from a very lively and active audience. It may not be surprising in a context were speakers and listeners are almost completely overlapping sets, but it still requires some extra effort when not everyone has a common ground. Hopefully, more events gathering psychological, formal and computational approaches to verse will take place in the near future. 